\title{
Rotational thromboelastometry and conventional coagulation tests in patients undergoing major cardiac or aortic surgery: a retrospective single-center cohort study
}

\author{
Cornelius Keyl $^{1}$ (1) $\cdot$ Albina Bashota $^{1} \cdot$ Friedhelm Beyersdorf $^{2} \cdot$ Dietmar Trenk $^{3}$
}

Accepted: 24 June 2021 / Published online: 7 July 2021

(c) The Author(s) 2021

\begin{abstract}
Algorithms for treatment of diffuse bleeding in cardiac surgery are based on intervention thresholds of coagulation tests, such as rotational thromboelastometry (ROTEM) or conventional laboratory tests. The relationship between these two approaches is unclear in patients with increased risk of coagulation abnormalities. We retrospectively analyzed the data of 248 patients undergoing major cardiac and/or aortic surgery. ROTEM and conventional laboratory tests were performed simultaneously after termination of cardiopulmonary bypass and protamine administration to investigate the extrinsic and intrinsic system, and to determine deficiencies in platelets and fibrinogen. We evaluated the association between ROTEM and conventional tests by linear regression analysis and compared the frequency of exceeding established thresholds for clinical intervention. Significant linear associations between ROTEM $10 \mathrm{~min}$ after the start of coagulation, and plasma fibrinogen concentration or platelet count (FIBTEM A10, $\mathrm{R}^{2}=0.67, \mathrm{p}<0.001$; EXTEM A10, $\mathrm{R}^{2}=0.47, \mathrm{p}<0.001$ ) were obtained. However, the $95 \%$ prediction intervals exceeded clinically useful ranges $(92-233 \mathrm{mg} / \mathrm{dL}$ fibrinogen at the intervention threshold of FIBTEM $\mathrm{A} 10=10 \mathrm{~mm} ; 14 \times 10^{3}-122 \times 10^{3} / \mu \mathrm{L}$ platelets at the intervention threshold of EXTEM A $\left.10=40 \mathrm{~mm}\right)$. The association between EXTEM and INR $\left(\mathrm{R}^{2}=0.23\right)$, and INTEM and $\mathrm{aPTT}\left(\mathrm{R}^{2}=0.095\right)$ was poor. The frequency of exceeding intervention thresholds and, consequently, of triggering treatment, varied markedly between ROTEM and conventional tests $(\mathrm{p}<0.001$ for all comparisons). The predictability of conventional coagulation test results by ROTEM is limited, thus hampering the interchangeability of methods in clinical practice.
\end{abstract}

Keywords Blood coagulation tests $\cdot$ Thromboelastography $\cdot$ Bleeding $\cdot$ Intraoperative complications $\cdot$ Cardiac surgical procedures

\section{Highlights}

Cornelius Keyl

cornelius.keyl@uniklinik-freiburg.de

1 Department of Anesthesiology, University Heart Center Freiburg - Bad Krozingen, Medical Faculty of the Albert-Ludwigs-University Freiburg, Suedring 15, 79189 Bad Krozingen, Germany

2 Department of Cardiovascular Surgery, University Heart Center Freiburg - Bad Krozingen, Medical Faculty of the Albert-Ludwigs-University, Freiburg, Germany

3 Department of Clinical Pharmacology, University Heart Center Freiburg - Bad Krozingen, Medical Faculty of the Albert-Ludwigs-University, Freiburg, Germany
- Rotational thromboelastometry (ROTEM) and conventional coagulation tests are considered as interchangeable to trigger therapeutic interventions in patients with increased risk of postoperative coagulation abnormalities

- We analyzed the relationship between methods in patients undergoing major cardiac and/or aortic surgery after termination of extracorporeal circulation and protamine administration

- We found a limited predictability of conventional coagulation tests by ROTEM, the $95 \%$ prediciton intervals exceeded clinically useful ranges

- The frequency of triggering therapeutic interventions varied significantly between methods 
- The evaluation of the therapeutic benefit of ROTEM or conventional tests for decision making is clinically important and should be subject of future research

\section{Introduction}

Rotational thromboelastometry (ROTEM) has been established as a point-of-care method to gain information on the coagulation profile in patients who are at high risk of diffuse bleeding in the intra- and postoperative period. Several algorithms for treatment of coagulation disorders, based on intervention thresholds of conventional coagulation tests or ROTEM, have been published in the last years [1-6]. A number of published investigations suggest a close interrelationship, expressed as statistically significant correlation or as linear regression model, between ROTEM and conventional coagulation parameters, such as plasma fibrinogen concentration, platelet count, international normalized ratio (INR) and activated partial thromboplastin time (aPTT) [7-13]. However, studies evaluating the association between ROTEM and conventional coagulation tests in patients undergoing major cardiac or aortic surgery with high risk of postoperative bleeding, as well as the impact of the coagulation assays on clinical decision-making, are lacking. Therefore, we retrospectively analyzed the data of patients who had undergone major cardiac and/or aortic surgery and who were predisposed to acquired coagulation disorders and bleeding complications. ROTEM and conventional coagulation tests were performed simultaneously after termination of extracorporeal circulation and heparin antagonization by protamine in all patients. Our study tested the hypothesis that there is a significant correlation between the results of ROTEM and conventional coagulation tests, and that plasma fibrinogen concentration, platelet count, INR, and aPTT can be predicted by ROTEM analyses. Furthermore, we tested the hypothesis, that the frequency of exceeding intervention thresholds, which serves as a trigger for therapeutic decisions in patients with ongoing diffuse bleeding, is comparable between ROTEM and conventional coagulation tests.

\section{Methods}

The study was performed as a retrospective single-center cohort study. We analyzed all data from patients 18 years of age or older undergoing major cardiac and/or aortic surgery (redo cardiac surgery, multiple valve surgery, surgery of native or prosthetic valve endocarditis, surgery of the aortic valve with simultaneous replacement of the ascending aorta, surgery of the aortic arch, and replacement of the thoracoabdominal aorta) between January 2015 and February 2019. The study protocol was approved by the local ethics committee who waived the need for obtaining informed consent of patients for this retrospective analysis (application ID 110/19, approved on July 2, 2019).

Data were retrieved from the electronic hospital information system (Patidok, Professional Clinical Software GmbH, Klagenfurt, Austria), and from the electronic patient database (Metavision, IMDsoft, Tel Aviv, Israel).

Routine clinical laboratory assessments were scheduled in all patients before surgery. Blood gas analyses and determination of electrolytes, hemoglobin, hematocrit and lactate were performed on a regular basis in all patients. ROTEM and conventional coagulation tests were performed simultaneously after termination of extracorporeal circulation and administration of protamine.

All coagulation assays (ROTEM and conventional coagulation tests) were performed by experienced laboratory staff in the clinical laboratory of our hospital. Blood samples were delivered by a pneumatic tube system and the results were communicated via the hospital intranet. Results of ROTEM were visualized in real time using the software "ROTEM live".

The conventional coagulation tests comprised the determination of platelet count by an automated flow cytometry assay (Sysmex XN 1000, Sysmex Norderstedt, Germany), fibrinogen using the Clauss method, prothrombin time, INR, and aPTT by routine laboratory assays (STA R Max, Stago, Düsseldorf, Germany).

ROTEM was performed using ROTEM delta (Werfen, München, Germany). The ROTEM tests comprised EXTEM (a tissue-activated test to analyze the clot formation via the extrinsic pathway), INTEM (a contact-activated test to analyze the clot formation via the intrinsic pathway) and FIBTEM (EXTEM plus the platelet inhibitor Cytochalasin $\mathrm{D}$ for analyzing the fibrinogen contribution to the clot). In addition, a contact-activated test with heparinase was used to exclude persisting heparin effects (HEPTEM) in all patients. Parameters used for analysis of coagulation were the time to the start of coagulation (CT) and the amplitude $10 \mathrm{~min}$ after the start of coagulation (A10).

The intervention thresholds for triggering therapeutic interventions in bleeding patients followed recommendations and algorithms published previously: extrinsic system, EXTEM CT $>80 \mathrm{~s}$, INR $>1.4$; intrinsic system, INTEM $\mathrm{CT}>240 \mathrm{~s}, \mathrm{aPTT}>50 \mathrm{~s}$; fibrinogen, FIBTEM A $10<10 \mathrm{~mm}$, plasma fibrinogen concentration $<200 \mathrm{md} / \mathrm{dL}$; platelets, EXTEM A $10<40 \mathrm{~mm}$, platelet count $<100 \times 10^{3} / \mu \mathrm{L}[1,4$, 14-16].

Fluid management comprised the administration of a balanced crystalloid solution, while artificial colloidal solutions were not used at that time. All patients received tranexamic acid following the dosage regimen published by Nutall et al. [17]. Patients experiencing increased bleeding before termination of extracorporeal circulation were 
initially transfused according to the clinical decision of the anesthesiologist. After determination of ROTEM and conventional coagulation tests, treatment with blood products (platelets, plasma) and coagulation factors (fibrinogen concentrate, prothrombin complex concentrate) was performed in patients with diffuse bleeding with respect to the clinical picture and the intervention thresholds of tests following the decision of the anesthesiologist and the surgical team.

\section{Statistical analysis}

Statistical analysis was performed using the software packages SPSS Statistics version 27 (IBM, Armonk, New York, USA) and Sigma Plot 13.0 (Systat Software, San Jose, CA, USA). A matrix plot visualizing missing data was created using the R software environment version 3.6.3 and the package "VIM".

Normal distribution of data was evaluated by visual assessment of the histograms and the probability plots (Q-Q plots). Normally distributed data are reported as mean \pm standard deviation, otherwise as median with 25 th and 75 th percentiles. Categorical variables were compared using Fisher's exact test for two-by-two tables. Tests were performed as two-sided tests with $\alpha=0.05$. The relationship between ROTEM and conventional coagulation tests was analyzed using linear regression analysis with the results of ROTEM as the predictor variable and conventional coagulation tests as the outcome variable. The normal distribution of residuals was evaluated using histograms and probability plots. Residual statistics were performed to detect outliers and influential cases. Cases with studentized residuals $>3$ or a critical Mahalanobis distance at $\mathrm{p}<0.001$ were examined in closer detail. Cases were excluded from analysis, if Cook's distance was greater than 1, and if they were obviously not plausible clinically. The lack of autocorrelation was tested using the Durbin-Watson test. The data were tested for heteroscedasticity by plotting studentized residuals against the predicted values and by the modified Preusch-Pagan test. In the case of heteroscedasticity, the $95 \%$ confidence interval for the regression coefficients was calculated using a bootstrapping process (corrected and accelerated confidence interval based on 1000 bootstrap samples).

The sample size was determined using the software G*Power (Department of Experimental Psychology, Heinrich-Heine-University, Düsseldorf, Germany). The minimally required sample size was calculated to detect a deviation of a single linear regression coefficient from zero, given $\mathrm{f}^{2}=0.05$, which corresponds to a small to medium effect size, with $\alpha=0.05$ and $\beta=0.1$. According to this assumption, we calculated a minimal sample size of 202 patients.

\section{Results}

The study cohort comprised 248 patients in total. The clinical characteristics of the patients are summarized in the Supplementary Table. A matrix plot of the available and missing data of ROTEM and conventional coagulation tests is provided in the Supplementary Figure.

The relationship between ROTEM and conventional coagulation tests is demonstrated in Fig. 1, the results of linear regression analysis are provided in Table 1.

\section{Relationship between EXTEM CT and INR}

Two patients were excluded due to missing values of INR, the results of 3 patients were identified as outliers and excluded from linear regression analysis.

Linear regression analysis revealed a significant relationship between EXTEM CT and INR, but the coefficient of determination was poor (Fig. 1A; Table 1). As the assumption of homoscedasticity was violated, the $95 \%$ confidence interval was calculated using bootstrapping.

\section{Relationship between INTEM CT and aPTT}

Four patients were excluded due to missing values (INTEM CT missing in two patients, aPTT missing in two patients), 13 patients were identified as outliers, mainly due to the finding that aPTT had reached the upper limit of the assay (160 s) in 9 of them. Linear regression analysis did not detect a clinically relevant ability of INTEM CT to predict aPTT (Fig. 1B; Table 1). As the assumption of homoscedasticity was violated, the $95 \%$ confidence interval was calculated using bootstrapping.

\section{Relationship between FIBTEM A10 and plasma fibrinogen concentration}

Seventeen patients were excluded due to missing values (fibrinogen concentration missing in 15 patients, FIBTEM A10 missing in two patients), one patient was identified as an outlier and excluded from regression analysis. The amplitude of FIBTEM A10 was significantly related to plasma fibrinogen concentration (Fig. 1C; Table 1). The intervention threshold in FIBTEM A10 $(=10 \mathrm{~mm})$ corresponded to a plasma fibrinogen level of $163 \mathrm{mg} / \mathrm{dL}$ fibrinogen with a $95 \%$ prediction interval of 92 to $233 \mathrm{mg} / \mathrm{dL}$ fibrinogen. 

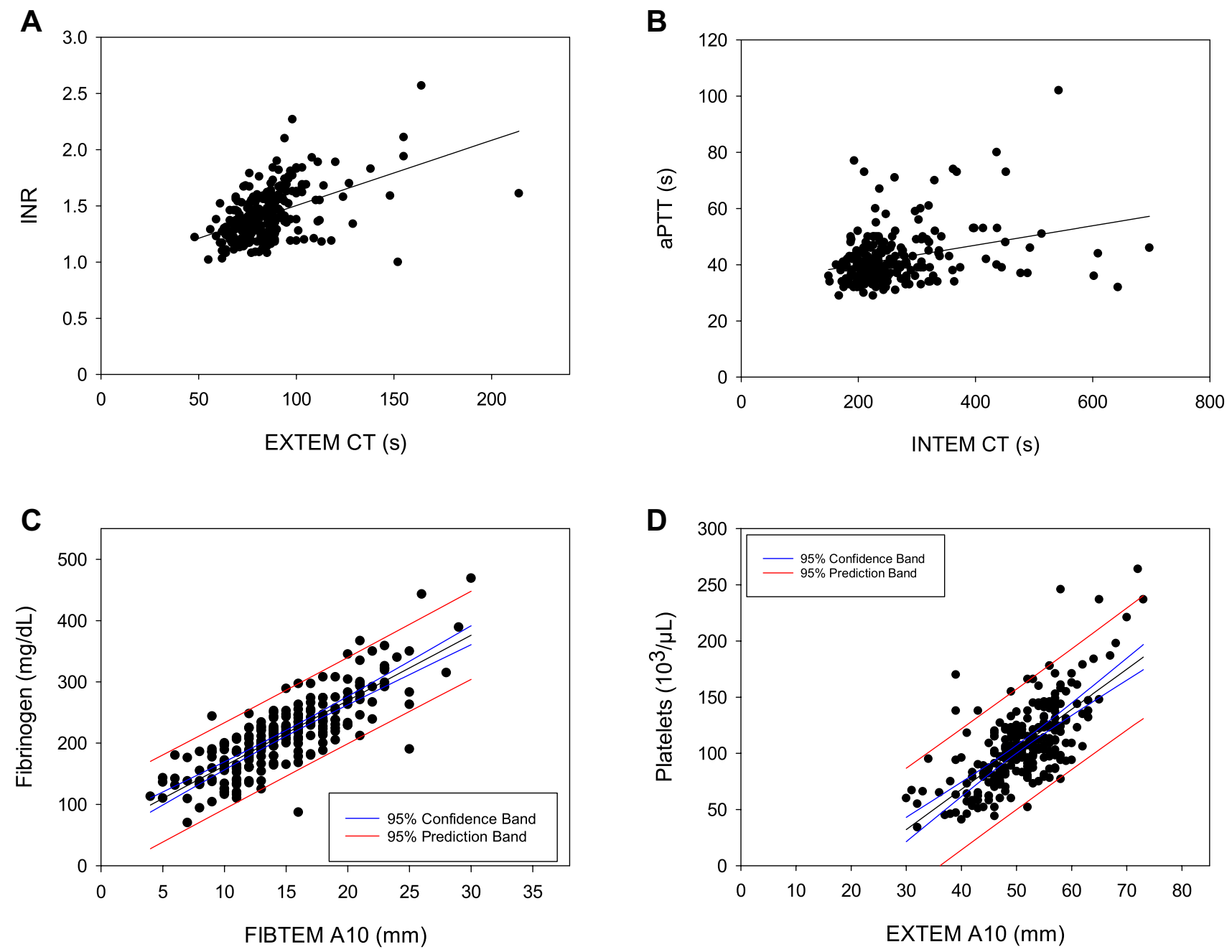

Fig. 1 Relationship between EXTEM CT and INR (A), INTEM CT und aPTT (B), FIBTEM A10 and plasma fibrinogen concentration (C), and EXTEM A10 and platelet count (D). The 95\% confidence intervals and $95 \%$ prediction intervals of linear regression are indi-

cated in graph C and D. Models A and B show heteroscedasticity, standard errors were estimated using bootstrapping. See Table 1 for details of regression analysis

Table 1 Details of linear regression analysis

\begin{tabular}{lllllll}
\hline Dependent variable & Independent variable & $\mathrm{R}^{2}$ & $\mathrm{p}$-value & Durbin-Watson & $\begin{array}{l}\text { Unstandardized coef- } \\
\text { ficient (95\% CI) }\end{array}$ & $\begin{array}{l}\text { Standardized } \\
\text { coefficient }\end{array}$ \\
\hline INR & EXTEM CT & 0.23 & $<0.001$ & 1.74 & $0.006(0.003 ; 0.009)$ & 0.48 \\
aPTT & INTEM CT & 0.095 & $<0.001$ & 1.76 & $0.035(0.013 ; 0.066)$ & 0.309 \\
$\begin{array}{l}\text { Plasma fibrinogen con- } \\
\quad \text { centration }\end{array}$ & FIBTEM A10 & 0.67 & $<0.001$ & 1.40 & $10.66(9.69 ; 11.63)$ & 0.82 \\
\begin{tabular}{l} 
Platelet count \\
\hline
\end{tabular} & EXTEM A10 & 0.47 & $<0.001$ & 2.21 & $3.57(3.09 ; 4.06)$ & 0.69 \\
\hline
\end{tabular}

\section{Relationship between EXTEM A10 and platelet count}

Eleven patients were excluded due to missing values (platelet count missing in 10 patients, EXTEM A10 missing in one patient), one patient was identified as an outlier and was excluded from linear regression analysis. Linear regression analysis revealed a significant relationship between EXTEM A10 and platelet count, but with a low coefficient of determination (Fig. 1D; Table 1). The 
intervention threshold of EXTEM A10 (=40 mm) was related to a platelet count of $68 \times 10^{3} / \mu \mathrm{L}$ with a $95 \%$ prediction interval of $14 \times 10^{3}$ to $122 \times 10^{3} / \mu \mathrm{L}$.

\section{Exceedance of intervention thresholds}

The intervention thresholds of tests and the results of crosstabulation analysis, comparing the frequency of data exceeding established intervention thresholds, are presented in Table 2. The frequency of exceeding the intervention threshold was significantly higher in ROTEM - tests evaluating the extrinsic and the intrinsic pathway compared to conventional coagulation assays. Regarding the evaluation of the extrinsic pathway of coagulation, 75 patients had pathological results in both, EXTEM CT and INR, whereas 61 patients (136 minus 75) exceeded the intervention threshold in EXTEM CT solely and 30 patients (105 minus 75) in INR solely. Regarding the assays evaluating the intrinsic pathway of coagulation, 30 patients had pathological results in both, INTEM CT and aPTT, whereas 81 patients exceeded the intervention threshold in INTEM CT solely and 8 patients in aPTT solely.

The frequency of exceeding the intervention threshold was significantly higher in FIBTEM A10 compared to the plasma fibrinogen concentration. Twenty-five patients exceeded the intervention threshold in both, FIBTEM A10 and plasma fibrinogen concentration, whereas a single patient exceeded the threshold in FIBTEM A10 solely, and 67 patients in fibrinogen concentration solely. Using $163 \mathrm{mg} / \mathrm{dL}$ instead of $200 \mathrm{mg} / \mathrm{dL}$ as the intervention threshold of plasma fibrinogen concentration, as predicted by the linear regression analysis, 45 patients (19.5\%), instead of 92 patients (39.8\%), exceeded this threshold. Eighteen patients were below the intervention threshold in both,
FIBTEM A10 and plasma fibrinogen concentration, whereas 8 patient exceeded the threshold in FIBTEM A10 solely and 27 patients in plasma fibrinogen concentration solely. Recalculating Fisher's exact test for two-by-two tables, a significant difference in triggering therapeutic interventions between FIBTEM A10 and plasma fibrinogen concentration was found $(\mathrm{p}<0.001)$.

The frequency of exceeding the intervention threshold was significantly higher in the platelet count compared to EXTEM A10. Fourteen patients exceeded the intervention threshold in both, EXTEM A10 and platelet count, whereas 2 patients exceeded the threshold in EXTEM A10 solely, and 93 patients in platelet count solely. When using $68 \times 10^{3}$ platelets $/ \mu \mathrm{L}$ instead of $100 \times 10^{3}$ platelets $/ \mu \mathrm{L}$ as the intervention threshold, as determined by linear regression analysis, 31 patients (13.1\%), instead of 107 patients $(45.1 \%)$, fell below this threshold. Eleven patients exceeded the threshold in both, EXTEM A10 and platelet count, whereas 5 patients exceeded the threshold in EXTEM A10 solely, and 20 patients in platelet count solely. Recalculating Fisher's exact test for two-by-two tables, a significant difference in triggering therapeutic interventions between EXTEM A10 and platelet count was found $(\mathrm{p}<0.001)$.

\section{The ratio HEPTEM CT/INTEM CT}

A HEPTEM CT / INTEM CT ratio $<0.66$ has been postulated to indicate a remaining heparin effect, i.e., an incomplete antagonization of heparin by protamine [18]. Fourteen patients had a HEPTEM CT / INTEM CT ratio $<0.66$ and received additional protamine. Overall, HEPTEM CT was not significantly shorter than INTEM CT (HEPTEM CT/ INTEM CT $=1.04[0.94 ; 1.18]$ ) in our patients, thus excluding a systematical bias due to a persisting heparin effect.

Table 2 Frequency of exceeding intervention thresholds in coagulation tests

\begin{tabular}{|c|c|c|c|c|c|}
\hline & & Intervention threshold & $\begin{array}{l}\text { Patients exceeding inter- } \\
\text { vention threshold, } \mathrm{n}(\%)\end{array}$ & $\mathrm{p}$ & $\begin{array}{l}\text { Patients exceeding interven- } \\
\text { tion thresholds in both tests, } \\
\mathrm{n}(\%)\end{array}$ \\
\hline \multirow[t]{2}{*}{ Extrinsic system $(n=246)$} & EXTEM CT & $80 \mathrm{~s}$ & $136(55.3)$ & $<0.001$ & $75(30.5)$ \\
\hline & INR & 1,4 & $105(42.7)$ & & \\
\hline \multirow[t]{2}{*}{ Intrinsic system $(n=244)$} & INTEM CT & $240 \mathrm{~s}$ & $111(45.5)$ & $<0.001$ & $30(12.3)$ \\
\hline & aPTT & $50 \mathrm{~s}$ & $38(15.6)$ & & \\
\hline \multirow[t]{2}{*}{ Fibrinogen $(n=231)$} & FIBTEM A10 & $10 \mathrm{~mm}$ & $26(11.3)$ & $<0.001$ & $25(10.8)$ \\
\hline & $\begin{array}{l}\text { Plasma fibrino- } \\
\text { gen concentra- } \\
\text { tion }\end{array}$ & $200 \mathrm{mg} / \mathrm{dL}$ & $92(39.8)$ & & \\
\hline \multirow[t]{2}{*}{ Platelets $(n=237)$} & EXTEM A10 & $40 \mathrm{~mm}$ & $16(6.8)$ & $<0.001$ & $14(5.9)$ \\
\hline & Platelet count & $100 \times 10^{3} / \mu \mathrm{L}$ & $107(45.1)$ & & \\
\hline
\end{tabular}




\section{Discussion}

The current study focused on patients undergoing major cardiac and/or aortic surgery with high risk of postoperative bleeding complications and coagulopathy. ROTEM and conventional coagulation analyses were determined simultaneously at a well-defined time point (after termination of extracorporeal circulation and antagonization of heparin by protamine), which is important for clinical decision-making. Our data demonstrate a limited interrelationship between ROTEM and conventional coagulation tests, which may cause substantial differences in therapeutic decisions depending on the methodology used.

It is well known that results of ROTEM and conventional tests are not directly comparable, as ROTEM measures the viscoelastic properties of whole blood under static conditions, while there is no contribution of platelet function or cellular elements in conventional coagulation tests due to pre-analytical removal of these components [19]. These methodological differences are, for instance, reflected by the varying congruence between coagulation tests based on the Clauss method for determination of plasma fibrinogen concentration and the clot firmness of ROTEM [13, 20]. The disagreement between these methods increased with supranormal values of plasma fibrinogen and after administration of fibrinogen concentrate [20]. In addition, several studies observed an effect of hematocrit on the amplitude of ROTEM, resulting in a higher correlation between ROTEM and plasma fibrinogen concentration with lower hematocrit [21,22]. Isovolemic hemodilution has different effect on coagulation tests: prothrombin time increased linearly to the dilution of coagulation factors, wheras aPTT, as well as EXTEM CT and INTEM CT, showed a nonlinear response [23].

Nevertheless, published diagnostic pathways postulate clinically significant relationships between EXTEM CT and INR, INTEM CT and APTT, FIBTEM A10 and plasma fibrinogen concentration, and EXTEM A10 and platelet count $[1,3,4,7-11]$, and there is an ongoing discussion whether the results of ROTEM and conventional coagulation tests may be regarded as interchangeable [19, 24].

We did not observe a clinically useful relationship between EXTEM CT and INR, and between INTEM $\mathrm{CT}$ and aPTT. Although there were statistically significant correlations between the results of ROTEM and the coagulation assays INR and aPTT, which have already been described in previous studies [7, 8, 10], our data demonstrate that a clinically applicable prediction of INR or aPTT by ROTEM is impossible, thus reinforcing comparable conclusions drawn by Haas et al. in a pediatric population [25]. Less than half of our patients with a pathological result of either ROTEM or INR exceeded the intervention thresholds in both tests. As a consequence, therapeutic implications differ substantially, depending on whether ROTEM or INR would be used to trigger treatment. Therefore, our data do not support approaches using ROTEM and INR as interchangeable indicators for the administration of prothrombin complex concentrate [1, 26]. Likewise, ROTEM and aPTT cannot be used as equivalent triggers for therapeutic interventions, as applied in a previous study [5]. Instead, our findings are comparable to the observations of Prakash et al., who reported a lack of interrelationship between INTEM CT and aPTT in patients who were anticoagulated with heparin for extracorporeal membrane oxygenation [27].

We determined a rather wide $95 \%$ prediction interval for the forecast of plasma fibrinogen concentration by FIBTEM. In addition, the plasma fibrinogen concentration associated with the intervention threshold of FIBTEM A10 differed markedly from previous assumptions. Previous studies postulated that the intervention threshold of FIBTEM A10 $=10 \mathrm{~mm}$ corresponds to a plasma fibrinogen concentration of approximately $200 \mathrm{mg} / \mathrm{dL}$ [4, 7], but we observed a substantially lower value ( $163 \mathrm{mg} / \mathrm{dL})$, comparable to the findings demonstrated by Mace et al. [11]. Using $163 \mathrm{mg} / \mathrm{dL}$ instead of $200 \mathrm{mg} / \mathrm{dL}$ as the intervention threshold of plasma fibrinogen concentration, 45 patients (19.5\%) instead of 92 patients $(39.8 \%)$ were below this threshold. Nevertheless, there was still a significant difference in triggering therapeutic interventions between ROTEM and plasma fibrinogen concentration. As a consequence, the clinical indication for fibrinogen substitution would have been inconsistent in most of the patients suspicious of fibrinogen deficiency, which again demonstrates the lack of interchangeability between the methods.

Even more pronounced inconsistencies were determined when analyzing the relationship between ROTEM and platelet count. As stated previously, viscoelastic test results are influenced by platelet function, which is regularly impaired after cardiac surgery with extracorporeal circulation [28, 29]. The impact of platelet count and platelet function on ROTEM amplitude has been discussed controversially: whereas some authors proposed the predictability of platelet count by ROTEM, others questioned this approach [30, 31].

The intervention threshold of platelet count in patients with diffuse bleeding varies markedly between publications, ranging from $50 \times 10^{3}$ to $100 \times 10^{3}$ platelets $/ \mu \mathrm{L}[1,3,15,32$, 33]. In accordance with previously published transfusion protocols, we defined EXTEM A $10<40 \mathrm{~mm}$ and a platelet count $<100 \times 10^{3}$ platelets $/ \mu \mathrm{L}$ as intervention thresholds for triggering the transfusion of platelets in patients with diffuse bleeding [4, 14, 15, 34]. In contrast, Kuiper et al. and Weber et al. proposed an association between EXTEM $\mathrm{A} 10=40 \mathrm{~mm}$ and a platelet count of $80 \times 10^{3}$ platelets $/ \mu \mathrm{L}$ $[1,32]$, which seems to be comparable with the observation 
of Ogawa et al. [7]. However, the platelet count predicted by EXTEM A10 $=40 \mathrm{~mm}$ was much lower in our patients, namely $68 \times 10^{3}$ platelets/ $\mu \mathrm{L}$. Furthermore, the large scattering of values, associated with a wide prediction interval, contradicted a clinically useful prediction of platelet count by ROTEM, as proposed previously [30], in our patients. As a consequence, the therapeutic decision to transfuse platelets would vary markedly in our patient cohort, depending on the test method used for decision support.

Up to now, it is unknown which laboratory methods, viscoelastic tests or conventional coagulation tests, or a combination of these assays, are preferable to monitor perioperative coagulation disorders $[19,24,35,36]$. An essential factor for initiating adequate treatment is the time lag between drawing the blood sample and receiving the test results. The results of ROTEM, visualized in real time via the hospital intranet, can be acknowledged after 10 to $15 \mathrm{~min}$, whereas results of conventional coagulation analyses are frequently delayed in time (20-30 min and longer), depending on local logistic factors. It may be hypothesized that the rapid availability of test results might be more important than the test assay used, as recently emphasized by Schmidt et al. [19].

\section{Limitations}

This retrospective analysis was not designed to evaluate the question as to whether viscoelastic tests, such as ROTEM, conventional coagulation tests, or a combination of these assays, are better suited for predicting postoperative bleeding, or for guiding transfusion therapy and replacement of coagulation factors. The replacement of blood products and coagulation factors in extensive cardiac and vascular surgery is a dynamic process, which, at best, is aimed to prevent the manifestation of severe coagulation and bleedings disorders and, at the same time, to avoid overtreatment and unnecessary use of resources. No standardized procedure depending solely on the results of ROTEM or the results of conventional coagulation analysis was mandatory in our clinical practice, and it was the responsibility of the anesthesiologist and the surgical team to take therapeutic decisions with respect to the clinical picture, as well as the test results.

\section{Conclusion}

In conclusion, our data demonstrate a limited predictability of conventional coagulation tests by ROTEM in clinical practice, thus hampering the interchangeability of methods. The frequency of triggering therapeutic interventions varies significantly between ROTEM and conventional coagulation assays. Controlled studies demonstrating the superiority of either approach in the treatment of bleeding patients are required, but are lacking up to now.

Supplementary Information The online version contains supplementary material available at https://doi.org/10.1007/s11239-021-02519-y.

Author contributions $\mathrm{AB}$ collected the data, contributed to data analysis, and corrected the manuscript. CK and DT performed the statistical analysis, prepared the figures and drafted the manuscript. FB discussed the results and contributed to the final manuscript. All authors read and approved the manuscript.

Funding Open Access funding enabled and organized by Projekt DEAL. This research did not receive any specific grant from funding agencies in the public, commercial, or not-for-profit sectors.

Data availability The data that support the findings of this study are available from the corresponding author upon reasonable request.

\section{Declarations}

Conflict of interest D. Trenk received consulting fees or advisory board fees and lecture fees paid by Amgen, AstraZeneca, Bayer, Boehringer Ingelheim KG, Bristol Myers Squibb, Daiichi Sankyo, Ferrer, Eli Lilly, and Pfizer. C. Keyl, F. Beyersdorf, and A. Bashota: no conflicts of interest to declare.

Ethical approval The study protocol was approved by the local ethics committee who waived the need for obtaining informed consent of patients for this retrospective analysis (application ID 110/19, approved on July 2, 2019).

Open Access This article is licensed under a Creative Commons Attribution 4.0 International License, which permits use, sharing, adaptation, distribution and reproduction in any medium or format, as long as you give appropriate credit to the original author(s) and the source, provide a link to the Creative Commons licence, and indicate if changes were made. The images or other third party material in this article are included in the article's Creative Commons licence, unless indicated otherwise in a credit line to the material. If material is not included in the article's Creative Commons licence and your intended use is not permitted by statutory regulation or exceeds the permitted use, you will need to obtain permission directly from the copyright holder. To view a copy of this licence, visit http://creativecommons.org/licenses/by/4.0/.

\section{References}

1. Weber CF, Gorlinger K, Meininger D, Herrmann E, Bingold T, Moritz A, Cohn LH, Zacharowski K (2012) Point-of-care testing: a prospective, randomized clinical trial of efficacy in coagulopathic cardiac surgery patients. Anesthesiology 117:531-547. https://doi.org/10.1097/ALN.0b013e318264c644

2. Fassl J, Matt P, Eckstein F, Filipovic M, Gregor M, Zenklusen U, Seeberger MD, Bolliger D (2013) Transfusion of allogeneic blood products in proximal aortic surgery with hypothermic circulatory arrest: effect of thromboelastometry-guided transfusion management. J Cardiothorac Vasc Anesth 27:1181-1188. https://doi.org/ 10.1053/j.jvca.2013.02.009

3. Karkouti K, Callum J, Wijeysundera DN, Rao V, Crowther M, Grocott HP, Pinto R, Scales DC, Investigators T (2016) 
Point-of-care hemostatic testing in cardiac surgery: a steppedwedge clustered randomized controlled trial. Circulation 134:1152-1162. https://doi.org/10.1161/CIRCULATIONAHA. 116.023956

4. Williams B, McNeil J, Crabbe A, Tanaka KA (2017) Practical use of thromboelastometry in the management of perioperative coagulopathy and bleeding. Transfus Med Rev 31:11-25. https:// doi.org/10.1016/j.tmrv.2016.08.005

5. Haensig M, Kempfert J, Kempfert PM, Girdauskas E, Borger MA, Lehmann S (2019) Thrombelastometry guided blood-component therapy after cardiac surgery: a randomized study. BMC Anesthesiol 19:201. https://doi.org/10.1186/s12871-019-0875-7

6. Monaco F, Nardelli P, Denaro G, De Luca M, Franco A, Bertoglio L, Castiglioni A, Zangrillo A (2020) First experience with a ROTEM-enhanced transfusion algorithm in patients undergoing aortic arch replacement with frozen elephant trunk technique. A theranostic approach to patient blood management. J Clin Anesth 66:109910. https://doi.org/10.1016/j.jclin ane.2020.109910

7. Ogawa S, Szlam F, Chen EP, Nishimura T, Kim H, Roback JD, Levy JH, Tanaka KA (2012) A comparative evaluation of rotation thromboelastometry and standard coagulation tests in hemodilution-induced coagulation changes after cardiac surgery. Transfusion 52:14-22. https://doi.org/10.1111/j.1537-2995.2011.03241.x

8. Theusinger OM, Schroder CM, Eismon J, Emmert MY, Seifert B, Spahn DR, Baulig W (2013) The influence of laboratory coagulation tests and clotting factor levels on Rotation Thromboelastometry $(\operatorname{ROTEM}(\mathrm{R}))$ during major surgery with hemorrhage. Anesth Analg 117:314-321. https://doi.org/10.1213/ANE.0b013e3182 9569ac

9. Olde Engberink RH, Kuiper GJ, Wetzels RJ, Nelemans PJ, Lance MD, Beckers EA, Henskens YM (2014) Rapid and correct prediction of thrombocytopenia and hypofibrinogenemia with rotational thromboelastometry in cardiac surgery. J Cardiothorac Vasc Anesth 28:210-216. https://doi.org/10.1053/j.jvca.2013.12.004

10. Schmidt DE, Holmstrom M, Majeed A, Naslin D, Wallen H, Agren A (2015) Detection of elevated INR by thromboelastometry and thromboelastography in warfarin treated patients and healthy controls. Thromb Res 135:1007-1011. https://doi.org/10.1016/j. thromres.2015.02.022

11. Mace H, Lightfoot N, McCluskey S, Selby R, Roy D, Timoumi T, Karkouti K (2016) Validity of thromboelastometry for rapid assessment of fibrinogen levels in heparinized samples during cardiac surgery: a retrospective, single-center, observational study. J Cardiothorac Vasc Anesth 30:90-95. https://doi.org/10.1053/j. jvca.2015.04.030

12. Ranucci M, Baryshnikova E (2016) Fibrinogen supplementation after cardiac surgery: insights from the Zero-Plasma trial (ZEPLAST). Br J Anaesth 116:618-623. https://doi.org/10.1093/ bja/aev539

13. Peng HT, Nascimento B, Beckett A (2018) Thromboelastography and thromboelastometry in assessment of fibrinogen deficiency and prediction for transfusion requirement: a descriptive review. Biomed Res Int 2018:7020539. https://doi.org/10.1155/2018/ 7020539

14. Gorlinger K, Dirkmann D, Hanke AA, Kamler M, Kottenberg E, Thielmann M, Jakob H, Peters J (2011) First-line therapy with coagulation factor concentrates combined with point-of-care coagulation testing is associated with decreased allogeneic blood transfusion in cardiovascular surgery: a retrospective, singlecenter cohort study. Anesthesiology 115:1179-1191. https://doi. org/10.1097/ALN.0b013e31823497dd

15. Bolliger D, Tanaka KA (2013) Roles of thrombelastography and thromboelastometry for patient blood management in cardiac surgery. Transfus Med Rev 27:213-220. https://doi.org/10.1016/j. tmrv.2013.08.004
16. Gorlinger K, Dirkmann D, Hanke AA (2013) Potential value of transfusion protocols in cardiac surgery. Curr Opin Anaesthesiol 26:230-243. https://doi.org/10.1097/ACO.0b013e32835ddca6

17. Nuttall GA, Gutierrez MC, Dewey JD, Johnson ME, Oyen LJ, Hanson AC, Oliver WC Jr (2008) A preliminary study of a new tranexamic acid dosing schedule for cardiac surgery. J Cardiothorac Vasc Anesth 22:230-235. https://doi.org/10.1053/j.jvca. 2007.12.016

18. Girdauskas E, Kempfert J, Kuntze T, Borger MA, Enders J, Fassl J, Falk V, Mohr FW (2010) Thromboelastometrically guided transfusion protocol during aortic surgery with circulatory arrest: a prospective, randomized trial. J Thorac Cardiovasc Surg 140(1117-24):e2. https://doi.org/10.1016/j.jtcvs.2010.04.043

19. Schmidt AE, Israel AK, Refaai MA (2019) The utility of thromboelastography to guide blood product transfusion. Am J Clin Pathol 152:407-422. https://doi.org/10.1093/ajcp/aqz074

20. Solomon C, Cadamuro J, Ziegler B, Schochl H, Varvenne M, Sorensen B, Hochleitner G, Rahe-Meyer N (2011) A comparison of fibrinogen measurement methods with fibrin clot elasticity assessed by thromboelastometry, before and after administration of fibrinogen concentrate in cardiac surgery patients. Transfusion 51:1695-1706. https://doi.org/10.1111/j.1537-2995.2011.03066.x

21. Ogawa S, Szlam F, Bolliger D, Nishimura T, Chen EP, Tanaka KA (2012) The impact of hematocrit on fibrin clot formation assessed by rotational thromboelastometry. Anesth Analg 115:16-21. https://doi.org/10.1213/ANE.0b013e31824d523b

22. Solomon C, Rahe-Meyer N, Schochl H, Ranucci M, Gorlinger K (2013) Effect of haematocrit on fibrin-based clot firmness in the FIBTEM test. Blood Transfus 11:412-418. https://doi.org/10. 2450/2012.0043-12

23. Weiss G, Lison S, Spannagl M, Heindl B (2010) Expressiveness of global coagulation parameters in dilutional coagulopathy. $\mathrm{Br} \mathrm{J}$ Anaesth 105:429-436. https://doi.org/10.1093/bja/aeq199

24. Avidan MS, Alcock EL, Da Fonseca J, Ponte J, Desai JB, Despotis GJ, Hunt BJ (2004) Comparison of structured use of routine laboratory tests or near-patient assessment with clinical judgement in the management of bleeding after cardiac surgery. Br J Anaesth 92:178-186. https://doi.org/10.1093/bja/aeh037

25. Haas T, Spielmann N, Mauch J, Madjdpour C, Speer O, Schmugge M, Weiss M (2012) Comparison of thromboelastometry $(\mathrm{ROTEM}(\mathrm{R}))$ with standard plasmatic coagulation testing in paediatric surgery. Br J Anaesth 108:36-41. https://doi.org/10. 1093/bja/aer342

26. Tanaka KA, Esper S, Bolliger D (2013) Perioperative factor concentrate therapy. Br J Anaesth 111(Suppl 1):i35-49. https://doi. org/10.1093/bja/aet380

27. Prakash S, Wiersema UF, Bihari S, Roxby D (2016) Discordance between ROTEM(R) clotting time and conventional tests during unfractionated heparin-based anticoagulation in intensive care patients on extracorporeal membrane oxygenation. Anaesth Intensive Care 44:85-92. https://doi.org/10.1177/0310057X16 04400113

28. Keyl C, Kmitta E, Kueri S, Zietak T, Trenk D (2011) Effects of aspirin and desmopressin on platelet reactivity in patients undergoing cardiac surgery with extracorporeal circulation. Thromb Haemost 105:113-121. https://doi.org/10.1160/TH10-07-0471

29. Ranucci M, Pistuddi V, Di Dedda U, Menicanti L, De Vincentiis C, Baryshnikova E (2019) Platelet function after cardiac surgery and its association with severe postoperative bleeding: the PLATFORM study. Platelets 30:908-914. https://doi.org/10.1080/09537 104.2018.1535706

30. Kuiper GJ, Henskens YM (2016) Rapid and correct prediction of thrombocytopenia and hypofibrinogenemia with rotational thromboelastometry in cardiac surgery reconsidered. J Cardiothorac Vasc Anesth 30:e55-e56. https://doi.org/10.1053/j.jvca.2016.04. 009 
31. Ranucci M, Di Dedda U, Baryshnikova E (2020) Platelet contribution to clot strength in thromboelastometry: count, function, or both? Platelets 31:88-93. https://doi.org/10.1080/09537104.2019. 1581920

32. Kuiper G, van Egmond LT, Henskens YMC, Roekaerts PM, Maessen JG, Ten Cate H, Buhre WF, Lance MD (2019) Shifts of transfusion demand in cardiac surgery after implementation of rotational thromboelastometry-guided transfusion protocols: analysis of the HEROES-CS (HEmostasis Registry of patiEntS in Cardiac Surgery) observational, prospective open cohort database. J Cardiothorac Vasc Anesth 33:307-317. https://doi.org/10.1053/j. jvca.2018.08.203

33. Task Force on Patient Blood Management for Adult Cardiac Surgery of the European Association for Cardio-Thoracic S, the European Association of Cardiothoracic A, Boer C, Meesters MI, Milojevic M, Benedetto U, Bolliger D, von Heymann C, Jeppsson A, Koster A, Osnabrugge RL, Ranucci M, Ravn HB, Vonk ABA, Wahba A, Pagano D (2018) 2017 EACTS/EACTA Guidelines on patient blood management for adult cardiac surgery. J Cardiothorac Vasc Anesth 32:88-120. https://doi.org/10.1053/j.jvca. 2017.06.026
34. Spahn DR, Bouillon B, Cerny V, Duranteau J, Filipescu D, Hunt BJ, Komadina R, Maegele M, Nardi G, Riddez L, Samama CM, Vincent JL, Rossaint R (2019) The European guideline on management of major bleeding and coagulopathy following trauma: fifth edition. Crit Care 23:98. https://doi.org/10.1186/ s13054-019-2347-3

35. Serraino GF, Murphy GJ (2017) Routine use of viscoelastic blood tests for diagnosis and treatment of coagulopathic bleeding in cardiac surgery: updated systematic review and meta-analysis. Br J Anaesth 118:823-833. https://doi.org/10.1093/bja/aex100

36. Wikkelso A, Wetterslev J, Moller AM, Afshari A (2017) Thromboelastography (TEG) or rotational thromboelastometry (ROTEM) to monitor haemostatic treatment in bleeding patients: a systematic review with meta-analysis and trial sequential analysis. Anaesthesia 72:519-531. https://doi.org/10.1111/anae.13765

Publisher's Note Springer Nature remains neutral with regard to jurisdictional claims in published maps and institutional affiliations. 